Check for updates

Cite this: RSC Adv., 2017, 7, 41339

\title{
Preparation of PTFE/PDMS superhydrophobic coating and its anti-icing performance
}

\author{
Min Ruan, (D) *a Yanlong Zhan, ${ }^{a}$ Yuansheng Wu, ${ }^{a}$ Xiangjie Wang, ${ }^{a}$ Wen $\mathrm{Li}{ }^{a}$ Yue Chen, ${ }^{a}$ \\ Maocai Wei, ${ }^{\text {b }}$ Xiuzhang Wang ${ }^{b}$ and Xiangyi Deng ${ }^{a}$
}

Superhydrophobic surfaces with anti-icing performance have been the focus of research but few studies have reported an effective strategy that met the requirements of excellent mechanical properties and durability. In this article, superhydrophobic coatings were prepared from a mixture of superfine polytetrafluoroethylene powder, polydimethylsiloxane and tetraethyl orthosilicate through spin-coating on aluminum substrate followed by solidification at $150{ }^{\circ} \mathrm{C}$. The static contact angle (CA) of the prepared coatings was as high as $163.6^{\circ}$. The surface morphology of the coatings was shown to be rough at micro/nano-scale with honeycomb microstructure, which allowed them to demonstrate excellent antiicing properties. The experiments show that the superhydrophobic surface exhibits excellent mechanical abrasion resistance after mechanical abrasion against 1000 grit SiC sandpaper for $1.0 \mathrm{~m}$ at the applied pressure of $14.4 \mathrm{kPa}$. The surfaces CA remained unchanged after one month and decreased to be $140.0^{\circ}$ after 34 subsequent icing/deicing experiments, which suggests good mechanical properties and durability of the prepared coatings. The present study may suggest a method for promising anti-icing applications in various fields.

Received 9th May 2017

Accepted 25th July 2017

DOI: $10.1039 / \mathrm{c} 7 \mathrm{ra0} 264 \mathrm{~b}$

rsc.li/rsc-advances materials with improved robustness and durability toward icing and deicing. ${ }^{21-23}$

Generally, superhydrophobicity comprises the combination of roughness at micro-nano scale and an intrinsic low-surfaceenergy of the materials used. For example, PDMS is frequently used for the preparation of SHSs because of its intrinsic CA of $110^{\circ}$ (i.e., CA of flat surface). However, flat PDMS does not exhibit a low value sliding angle, and thus various filler materials should be mixed with PDMS in order to make it rough and achieve superhydrophobic surface. ${ }^{16,24-29}$ Manca et al. used SU-8 masters to fabricate micropillars onto PDMS sample and then fabricated nanoposts on their top and enhanced their fluorination rate by using inductively coupled plasma (ICP) technique. ${ }^{30}$ Xiu et al. studied the superhydrophobicity PDMS/PTFE composites applied on top of Si wafer via dip-coating, barcoating, or spin-coating. ${ }^{31}$ Tropmann et al. used PDMS/PTFE composite particles and $\mathrm{CF}_{4} / \mathrm{O}_{2}$ plasma treatment for isotropic superhydrophobic PDMS surface. ${ }^{\mathbf{1 6}}$

In this study, aluminum plates were used as the substrates because of their easy availability, low price and wide applications in transportation, packaging, construction, electrical transmission lines and so on. Alumina-based SHSs were produced using PDMS (polydimethylsiloxane), TEOS (tetraethyl orthosilicate), DOTL (dioctyldilauryltin) and PTFE (polytetrafluroethylene) as precursors. To optimize the materials' water-repellent properties, we studied the influence of the amount of PTFE. The antiicing performance and mechanical property of optimized samples was investigated, demonstrating a good level of stability.
${ }^{a}$ Institute of Materials Science and Engineering, Hubei Key Laboratory of Mine Environmental Pollution Control \& Remediation, Hubei Polytechnic University, Huangshi, China. E-mail: huangxi090108@gmail.com; Tel: +86-714-6348386 ${ }^{b}$ Advanced Materials Research Institute, Hubei Normal University, China 


\section{Materials and methods}

Aluminum plates with purity of $99.6 \%$ and anhydrous ethanol were purchased from Tianjin Tianli chemical reagents company. PDMS with viscosity of $500 \mathrm{~mm}^{2} \mathrm{~s}^{-1}$ was purchased from Tianjin Bodi chemical company. TEOS was purchased from Chengdu Kelong chemical company. DOTL was purchased from Sa'en chemical technological company. PTFE was purchased from DuPont company. The aluminum plates $(10 \times$ $10 \times 0.1 \mathrm{~mm}^{3}$ in size) were used as substrates. They were first degreased using 2000\# metallographic abrasive paper and cleaned ultrasonically, subsequently with alcohol and deionized water for $5 \mathrm{~min}$, after which dried in air at $60{ }^{\circ} \mathrm{C}$ for $0.5 \mathrm{~h}$.

The values of CA were measured on a Krüss DSA 100 contactangle goniometer (Krüss GmbH, Germany) following standard procedures previously described by others. ${ }^{14,21}$ The surface morphology of the samples was observed using scanning electron microscopy (SEM; FEI Quanta 200). FTIR spectral data were measured with the $\mathrm{KBr} / \mathrm{PDMS}$ or $\mathrm{KBr} / \mathrm{PDMS} / \mathrm{PTFE}$ disks (prepared with $0.1998 \mathrm{~g}$ of powdered $\mathrm{KBr}$ and $0.0002 \mathrm{~g}$ of PDMS or PDMS/PTFE as ref. 32) with Bruker Tensor 27. An atomic force microscopic (AFM; MFP-3D Origin AFM from Oxford) was operated in the contact mode for visualization of the surfaces.

PDMS and PTFE were the main components of the raw material, while DOTL and TEOS were used as modifiers and to facilitate solidification processes. First, DOTL was added to PDMS to form a suspension, then different amounts of PTFE powder were added to the above suspension followed by the addition of TEOS. The weight of PDMS, TEOS, and DOTL was 3, 1.2 , and $0.3 \mathrm{~g}$, respectively. Then the as-prepared mixture was deposited on the prepared aluminum substrate by means of spin-coater. After coating, the samples were dried at $150{ }^{\circ} \mathrm{C}$ in oven.

The mechanical abrasion resistance of the prepared SHS sample was tested against 1000 grit SiC sandpaper for $1.0 \mathrm{~m}$ at the applied pressure of $14.4 \mathrm{kPa}$. The sample was placed facedown to sandpaper and moved for $0.25 \mathrm{~m}$ along one direction, then the sample was rotated by $90^{\circ}$ (face to the sandpaper) and then moved for $0.25 \mathrm{~m}$ along one direction. This process was defined as one abrasion cycle, and repeated the cycle for four times to complete the abrasion test.

Furthermore, the anti-icing behavior of the samples was tested with a home-made icing monitoring system whose schematic diagram is shown in Fig. 1. The system consists of water-cooling system (water-cooling power of about $5 \mathrm{~W}$ ), semiconductor material refrigeration slice (cooling power of about $25.2 \mathrm{~W})$, temperature sensor, sample stage $\left(5 \times 3 \mathrm{~cm}^{2}\right)$, camera and controlling computer. The temperature of the sample surface and icing process can be real-time monitored by the home-made simple system. The icing/deicing process was also implemented by the system. The specific implementation procedures would be described subsequently.

\section{Results and discussion}

To investigate the effect of the amount of PTFE on the sample superhydrophobicity, 0, 0.2, 0.4, 0.6, 0.8 and $1.0 \mathrm{~g}$ PTFE was

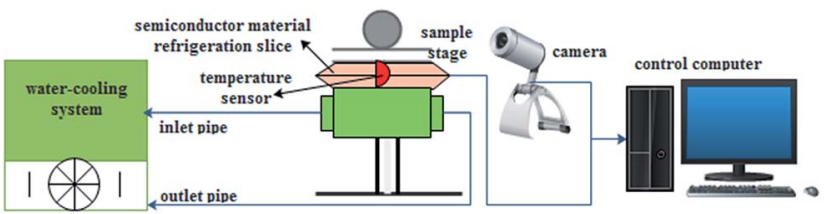

Fig. 1 Scheme of the home-made icing monitoring system used in the present study.

mixed with PDMS, TEOS, and DOTL. Fig. 2 shows how the amount of added PTFE influenced the CA value of produced coating. It is clearly seen that PTFE played a significant role in the superhydrophobicity of the samples. While the value of PTFE-free sample was $129.0^{\circ}$, it could reach $163.6^{\circ}$ (with the addition of $0.6 \mathrm{~g}$ PTFE) of the optimized sample. It was also seen in Fig. 2 that when more PTFE was added into the spin-coated mixture, no further increase of $\mathrm{CA}$ was achieved, and the highest CA value was obtained at the weight ration of the initial materials of PDMS : TEOS : DOTL : PTFE $=10: 4: 1: 2$. It may because of the microscopic structure of the surface, when there was no or little PTFE, the surface was too smooth to "trap" air between the mastoid structure. On the other hand, if there was too much PTFE grain, they could fill the gap between the micronano structure to some extent which would decrease the roughness of the surface and the CA.

Fig. 3a-c present SEM surface images of as-prepared coatings without PTFE added (a), with $0.6 \mathrm{~g}$ of PTFE (b), and with $1.0 \mathrm{~g}$ of PTFE (c). For comparison, panel (d) exhibits the surface image of the sample in panel (b) that was subjected to 34 icing/deicing experiments. It is seen in Fig. 3a, the sample spin-coated without PTFE is smooth, while those prepared with addition of PTFE are rough at micro- and nano-scale with honeycomb-shaped surface roughness as shown in Fig. $3 b-d$. Water droplets are expected to form a larger liquid-solid contact area ${ }^{12,33}$ with the smooth surface whose CA is $129.0^{\circ}$ in Fig. 3 a. At the same time,

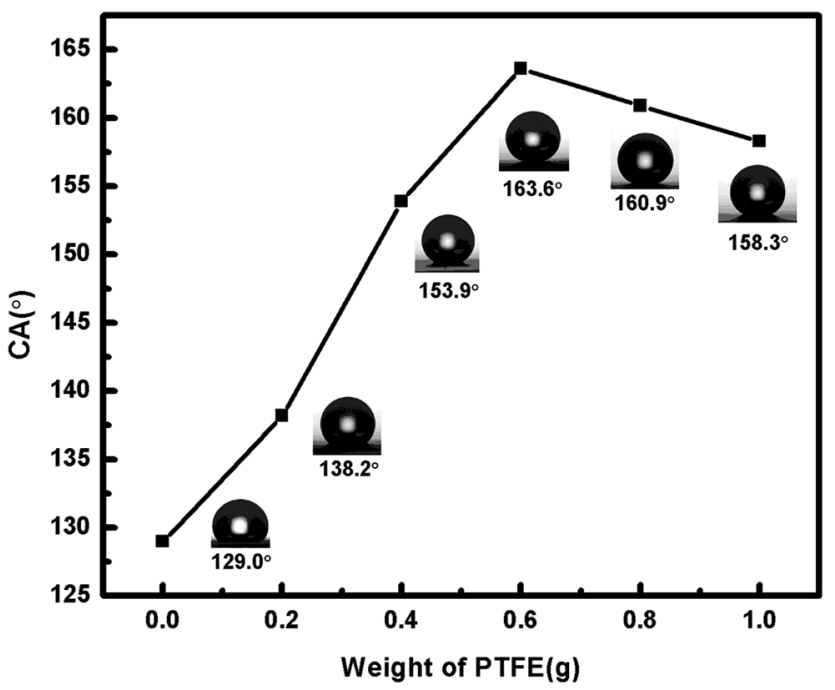

Fig. 2 CA values of coatings prepared with different amount of PTFE $(0,0.2,0.4,0.6,0.8,1.0 \mathrm{~g})$ added to spin-coated mixture. 


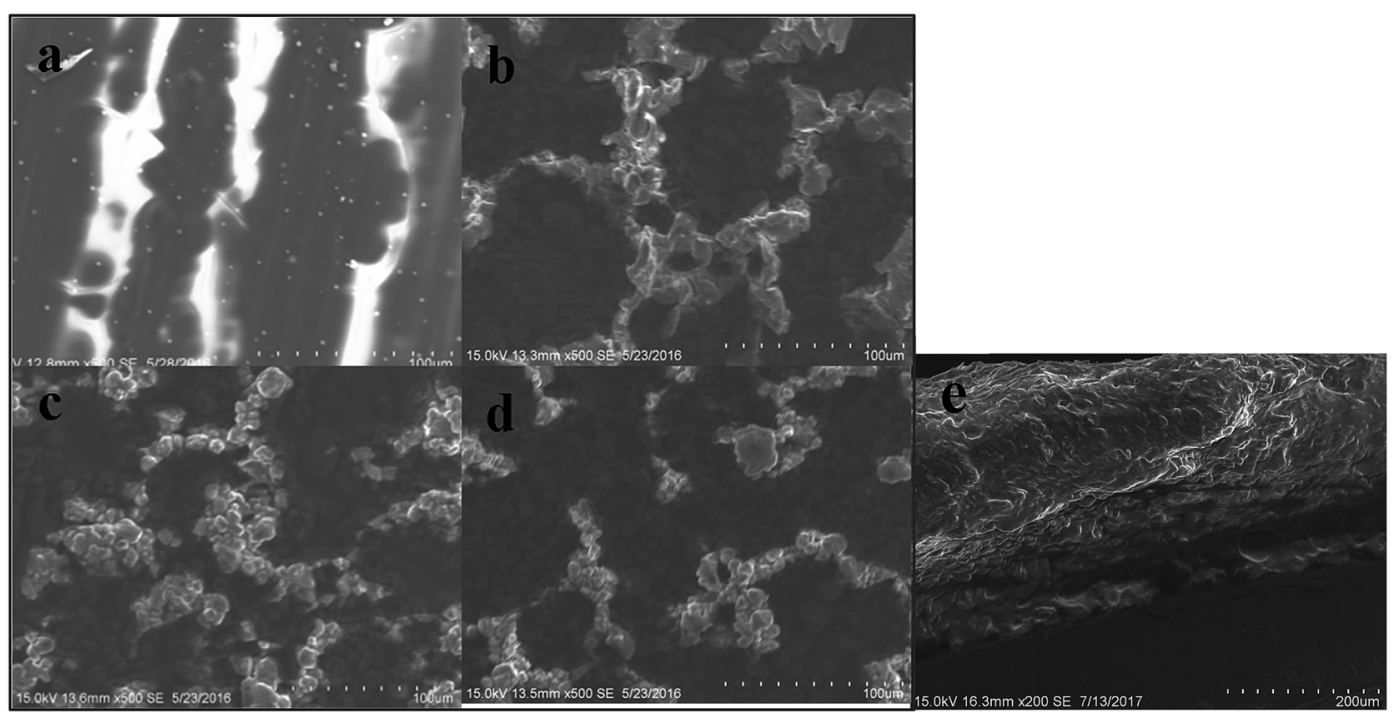

Fig. 3 SEM surface images of coatings prepared with different amounts of PTFE added to spin-coated mixture: (a) PTFE-free coating, (b) with $0.6 \mathrm{~g}$ of PTFE, (c) with $1.0 \mathrm{~g}$. For comparison, panel (d) demonstrates surface from panel (b) subjected to icing/deicing 34 times. (e) is the SEM image of vertical surface of SHS coating.

water drops are expected to contact only a small fraction of solid surface (mainly tops of rough structures) on the SHSs with the CA of $163.6^{\circ}$ and $158.3^{\circ}$ (for which 0.6 and $1.0 \mathrm{~g}$ of PTFE was used in spin-coated suspensions).

Thus, the roughness (and wettability) of prepared coatings could be controlled through the amount of PTFE incorporated into the coating, as seen in Fig. 2 and $3 \mathrm{~b}$ and c. The optimized sample (shown in Fig. 3b) had its weight ratio of PDMS : TEOS : PTFE : DOTL equal to $10: 4: 1: 2$. Importantly, its morphology was basically unchanged after 34 icing/deicing cycles, showing that only a small fraction of surface rough structures were damaged/removed from the surface during icing/deicing (compare Fig. 3b and d). Fig. 3e shows the SEM image of the vertical surface of the SHS coating with the thickness of the about $205 \mu \mathrm{m}$.

Fig. 4a shows the AFM image of the optimized sample with the weight ratio of PDMS : TEOS : PTFE $:$ DOTL $=10: 4: 1: 2$ and its root-mean-square (rms) roughness is $44.8 \mathrm{~nm}$. It shows that the SHS surface has deep groove and is plain regionally with CA of $163.6^{\circ}$. After the abrasion test of the prepared SHS surface, a porous morphology can be seen in the AFM image with its roughness of $23.3 \mathrm{~nm}$ and $\mathrm{CA}$ of $140.0^{\circ}$ as shown in Fig. $4 \mathrm{~b}$. It seems that the CA of the surface is dependent on the roughness. Besides roughness, the superhydrophobicity also has a relationship with the chemical compositions of the surface. FTIR is used to investigate the influence of it.

Fig. 5 compares FTIR spectra of as-prepared PTEF-free PDMS coating (dotted line) and of SHS coating with $0.6 \mathrm{~g}$ of PTFE (red solid line). The new very strong bands at $1262 \mathrm{~cm}^{-1}$ and $1094 \mathrm{~cm}^{-1}$ corresponding to the asymmetric and symmetric stretching of C-F bond in the SHS sample spectrum (red solid line in Fig. 5). ${ }^{6}$ The spectra clearly indicate the presence of PTFE on the SHS sample surface, which promises the interaction of PTFE with aluminum surface.

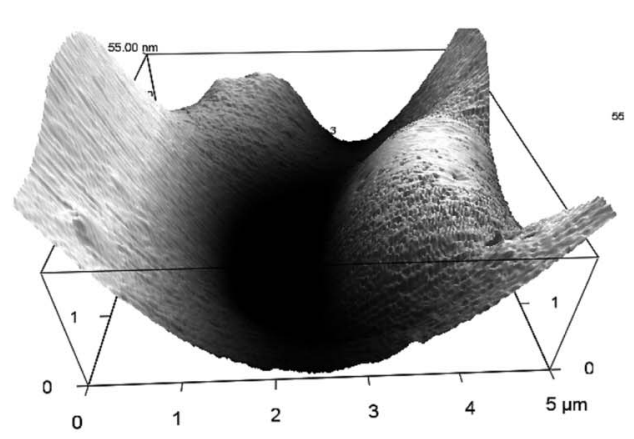

(a)

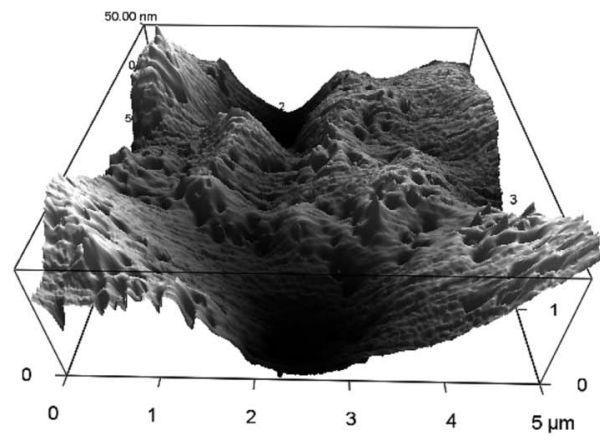

(b)

Fig. 4 The AFM images of (a) the prepared SHS surface and (b) the surface after abrasion test. 


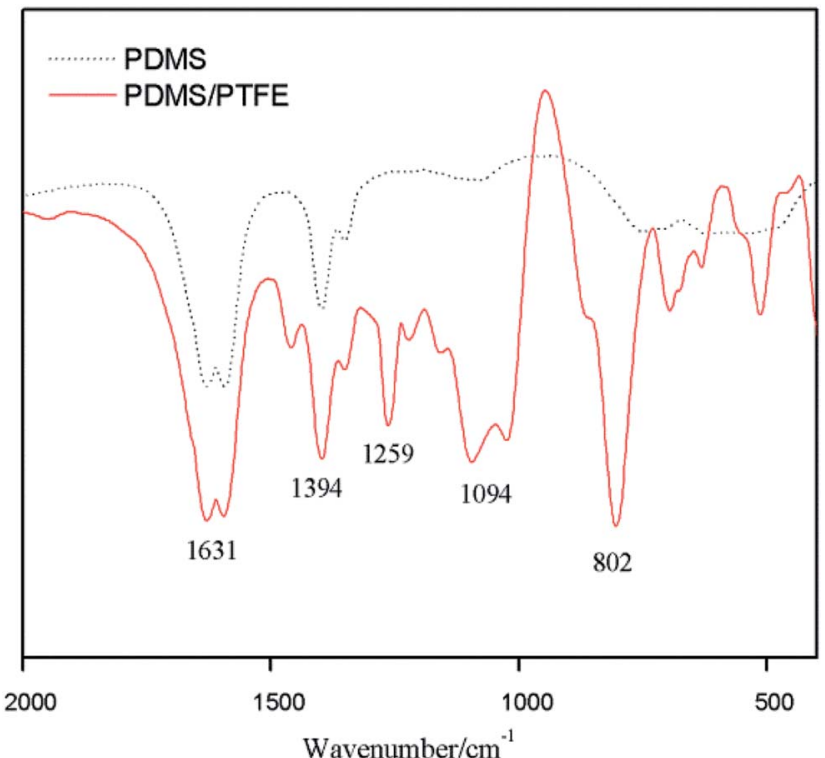

Fig. 5 FTIR spectra of PDMS coating (dot line) and PDMS/PTFE composite coating with $0.6 \mathrm{~g}$ of PTFE (red solid line).

Fig. 6 exhibits how a water droplet ( $4 \mu \mathrm{l}$ in volume) freezes on the above described optimized SHS sample, an untreated aluminum plate with a similar water droplet being presented for comparison. The two samples were first put horizontally on the sample stage of the home-made icing monitoring system in air with relative humidity of about $60 \%$, after which the surface temperature of the sample stage was decreased from $13.3^{\circ} \mathrm{C}$ to $-6^{\circ} \mathrm{C}$ with a cooling rate of $12{ }^{\circ} \mathrm{C} \mathrm{min}{ }^{-1}$. Fig. $6 \mathrm{a}$ and $\mathrm{b}$ present the initial images of the water droplets on the SHS and noncoated bare aluminum surfaces, respectively. In agreement with previous reports by others, ${ }^{34}$ the water droplet was spread more and tended to wet the bare aluminum surface, while it attained a sphere-like shape on the SHS surface.

Because of a high cooling rate applied $\left(12{ }^{\circ} \mathrm{C} \mathrm{min}^{-1}\right)$ and small volume of water droplets, the temperature of the surface decreased rapidly. As a result, the droplet placed onto the noncoated aluminum demonstrated ice nucleation inside and was completely frozen just within $2 \mathrm{~s}$ (already $37 \mathrm{~s}$ after the experiment began), when the surface temperature only reached $-0.5{ }^{\circ} \mathrm{C}$. This is associated with the light region disappearing in the middle of the right-hand-side droplet in Fig. 6d, indication git was no longer transparent but opaque and solid. Meanwhile, due to the low temperature and relatively high humidity in the atmosphere, the initially spherical droplet on the SHS spread a little at $-0.5{ }^{\circ} \mathrm{C}$, still remaining transparent (Fig. 6c). Ice nucleation only began in it after as long as $82 \mathrm{~s}$ when the temperature reached $-3.6{ }^{\circ} \mathrm{C}$ and the relative humidity was $\sim 52 \%$, as shown in Fig. 6e. After initial ice nuclei formed, it took about $5 \mathrm{~s}$ for the water droplet to freeze completely on the SHS surface, as well seen in Fig. 6i where a fully opaque droplet is presented.

Thus, a delay (of $\sim 50 \mathrm{~s}$ ) of water drop freezing was achieved on the prepared SHS sample compared with the untreated surface. It is worth noting, however, that though the two

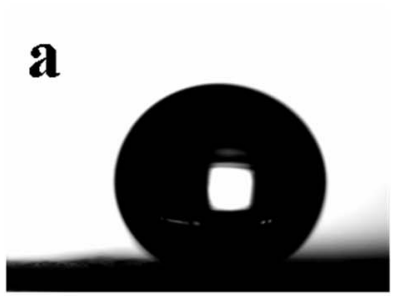

b $\quad$ T:0s
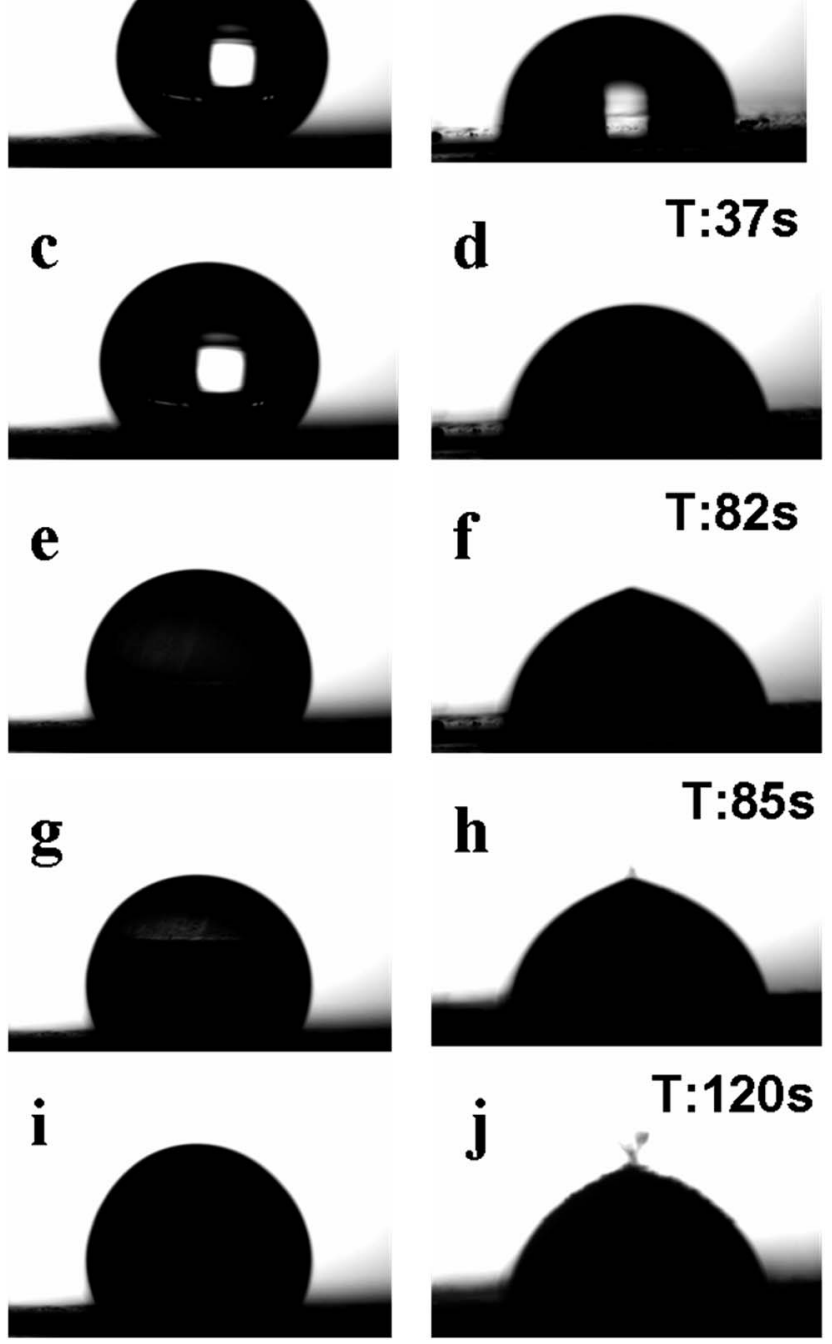

Fig. 6 Water drops freezing on SHS sample (left) and untreated aluminum plate (right). (a and b) Initial water drops just placed onto surfaces at $13.3^{\circ} \mathrm{C}$. (c) The water drop spread a little on SHS at $-0.5^{\circ} \mathrm{C}$. (d) Ice nucleation starts at $-0.5^{\circ} \mathrm{C}$ on untreated aluminum. (e) Ice nucleation starts at $-3.6{ }^{\circ} \mathrm{C}$ on SHS sample. (f and h) Completely frozen droplet on untreated aluminum. (g) Freezing proceeds on SHS at $-3.6^{\circ} \mathrm{C}$. (i and j) Iced droplets at $-6.0^{\circ} \mathrm{C}$.

surfaces were placed on the same freezing stage and were cooled down under similar conditions, the stage temperature was constantly decreasing, and while the droplet on the uncoated aluminum froze at $-0.5^{\circ} \mathrm{C}$, its counterpart on the SHS got completely solidified at $-3.5{ }^{\circ} \mathrm{C}$. This implies that the freezing delay on the SHS is believed to be much longer provided that the stage temperature is kept constant at $-0.5^{\circ} \mathrm{C}$.

The observed delay in freezing of water on SHS agrees well with previous reports by others and was explained in several previous reports. ${ }^{34}$ The actual water-solid contact area of water droplets resting on SHS (which are known to be in the CassieBaxter wetting mode) is known to be quite small, on the order of $10 \%$ or lower, ${ }^{34}$ the remaining contact area being the water-air 


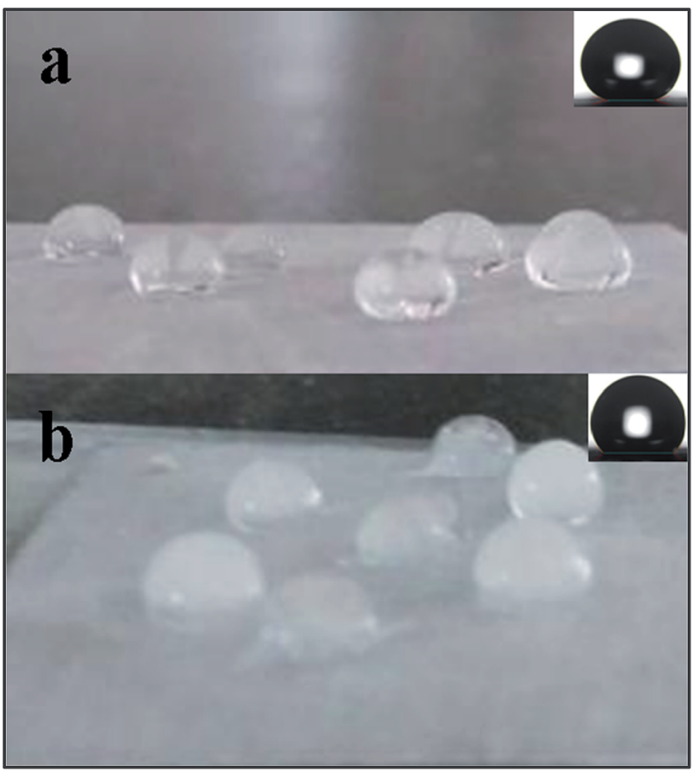

Fig. 7 (a) Initial state of water drops freezing on the optimized SHS sample, the inserted figure represents the water droplet on the surface with CA of $163.6^{\circ}$. (b) Frozen state after 34 times icing/deicing cycles, the inset being a water image with CA of $140.0^{\circ}$.

interface. Air is known as a good thermal insulator, and thus it slows down any energy exchange between water drops and SHS. Consequently, the heat transfer via the liquid-solid contact interface on SHSs is lower when compared with that on flat noncoated aluminum (with no air pockets trapped into surface roughness). ${ }^{34}$

The icing/deicing experiments were carried out using the same above mentioned system. Seven to ten water drops (of $\sim 4 \mu$ in volume) were placed onto the optimized SHS randomly, after which the stage temperature was decreased from room temperature to $-6{ }^{\circ} \mathrm{C}$. Upon freezing, all the iced drops were then removed by applying external mechanical force (shear stress). Due to the low temperature, the sample was frozen and fixed on the sample stage, we used a pair of tweezers to remove the frozen drops by a external force in parallel to the sample. This process must be completed rapidly, because the frozen water droplets are very small and they will melt in short time. After deicing, the sample was dried in oven at $60{ }^{\circ} \mathrm{C}$ for $3 \mathrm{~min}$, after which the same procedures were repeated again.

Fig. 7a and b show liquid water drops on the as-prepared SHS sample (a) and similar drops frozen on the same sample subjected to 34 icing/deicing cycles (b). It is seen that all water drops were spherical on the as-prepared sample (a). Meanwhile, the frozen drops in Fig. $7 \mathrm{~b}$ are seen to wet the surface, thus giving rise to larger ice adhesion strength on the sample that was iced/deiced 34 times. As was previously reported by others, ${ }^{17,21-23}$ SHSs tend to be gradually damaged by icing/ deicing. This is also somewhat supported by Fig. $3 \mathrm{~b}$ and $\mathrm{d}$ where the surface of the sample iced/deiced 34 times shows somewhat lower roughness. This should result in slightly higher water/solid contact area when water drops are placed on such a surface (compare insets in Fig. 7a and b).

\section{Conclusions}

PTFE powder is shown to be effective to obtain superhydrophobic coatings on aluminum substrate, with water contact angle (CA) values reaching as high as $163.6^{\circ}$. In this study, PTFE was dispersed in composite coating at the ratio of PDMS : TEOS : DOTL : PTFE = 10:4:1:2. The prepared coatings were stable even after one month. The surface morphology was found to be rough honeycomb structures at micro-nano scales. After as many as 34 icing/deicing cycles performed in a home-made icing monitoring system, the surface rough structures were observed to experience just minor changes, which led to CA of $140^{\circ}$.

\section{Conflicts of interest}

There are no conflicts to declare.

\section{Acknowledgements}

This work was financially supported by the Provincial Key Program of Natural Science Foundation of Hubei Province, China (T201423, T201626) and NSFC (51405146, 51372077).

\section{References}

1 Y. Cao, Z. Wu, Y. Su and Z. Xu, Progr. Aero. Sci., 2015, 74, 6280.

2 H. Habibi, L. Cheng, H. Zheng, V. Kappatos, C. Selcuk and T.-H. Gan, Renewable Energy, 2015, 83, 859-870.

3 S. A. Kulinich, M. Honda, A. L. Zhu, A. G. Rozhin and X. W. Du, Soft Matter, 2015, 11, 856-861.

4 C. Antonini, M. Innocenti, T. Horn, M. Marengo and A. Amirfazli, Cold Reg. Sci. Technol., 2011, 67, 58-67.

5 H. Zheng, S. Chang and Y. Zhao, Progr. Chem., 2017, 29, 102118.

6 N. Dalili, A. Edrisy and R. Carriveau, Renewable Sustainable Energy Rev., 2009, 13, 428-438.

7 L. Zheng, Z. Li, S. Bourdo, K. R. Khedir, M. P. Asar, C. C. Ryerson and A. S. Biris, Langmuir, 2011, 27, 9936-9943.

8 J.-H. Li, Q. Liu, Y.-L. Wang, R.-R. Chen, K. Takahashi, R.-M. Li, L.-H. Liu and J. Wang, J. Electrochem. Soc., 2016, 163, C213-C220.

9 Y. Lai, Y. Tang, J. Gong, D. Gong, L. Chi, C. Lin and Z. Chen, J. Mater. Chem., 2012, 22, 7420-7426.

10 J.-H. Oh, T.-J. Ko, M.-W. Moon and C. H. Park, RSC Adv., 2017, 7, 25597-25604.

11 L. Cao, A. K. Jones, V. K. Sikka, J. Wu and D. Gao, Langmuir, 2009, 25, 12444-12448.

12 M. Ruan, W. Li, B. Wang, B. Deng, F. Ma and Z. Yu, Langmuir, 2013, 29, 8482-8491.

13 N. Puretskiy, J. Chanda, G. Stoychev, A. Synytska and L. Ionov, Adv. Mater. Interfaces, 2015, 2, 1500124.

14 S. A. Kulinich and M. Farzaneh, Appl. Surf. Sci., 2009, 255, 8153-8157.

15 P. Guo, Y. Zheng, M. Wen, C. Song, Y. Lin and L. Jiang, Adv. Mater., 2012, 24, 2642-2648. 
16 A. Tropmann, L. Tanguy, P. Koltay, R. Zengerle and L. Riegger, Langmuir, 2012, 28, 8292-8295.

17 Y. Wang, J. Xue, Q. Wang, Q. Chen and J. Ding, ACS Appl. Mater. Interfaces, 2013, 5, 3370-3381.

18 Y. Tang, Q. Zhang, X. Zhan and F. Chen, Soft Matter, 2015, 11, 4540-4550.

19 L. Wang, Q. Gong, S. Zhan, L. Jiang and Y. Zheng, Adv. Mater., 2016, 28, 7729-7735.

20 C. Zhu, S. Liu, Y. Shen, J. Tao, G. Wang and L. Pan, Surf. Coat. Technol., 2017, 309, 703-708.

21 S. Farhadi, M. Farzaneh and S. Kulinich, Appl. Surf. Sci., 2011, 257, 6264-6269.

22 S. Kulinich, S. Farhadi, K. Nose and X. Du, Langmuir, 2010, 27, 25-29.

23 A. Lazauskas, A. Guobienè, I. Prosyčevas, V. Baltrušaitis, V. Grigaliūnas, P. Narmontas and J. Baltrusaitis, Mater. Charact., 2013, 82, 9-16.

24 K. Li, X. Zeng, H. Li, X. Lai, C. Ye and H. Xie, Appl. Surf. Sci., 2013, 279, 458-463.
25 Z.-Y. Deng, W. Wang, L.-H. Mao, C.-F. Wang and S. Chen, J. Mater. Chem. A, 2014, 2, 4178.

26 X. Liu, Y. Xu, K. Ben, Z. Chen, Y. Wang and Z. Guan, Appl. Surf. Sci., 2015, 339, 94-101.

27 C. Yang, F. Wang, W. Li, J. Ou, C. Li and A. Amirfazli, Appl. Phys. A: Mater. Sci. Process., 2016, 122, 1.

28 X. Liu, Y. Xu, Z. Chen, K. Ben and Z. Guan, RSC Adv., 2015, 5, 1315-1318.

29 E. J. Park, J. K. Sim, M.-G. Jeong, H. O. Seo and Y. D. Kim, RSC Adv., 2013, 3, 12571-12576.

30 M. Manca, B. Cortese, I. Viola, A. S. Aricò, R. Cingolani and G. Gigli, Langmuir, 2008, 24, 1833-1843.

31 Y. Xiu, L. B. Zhu, D. W. Hess and C. P. Wong, Nano Lett., 2007, 7, 3388-3393.

32 T. H. Dakhakhni, G. A. Raouf and S. Y. Qusti, Eur. Biophys. J., 2016, 45, 311-320.

33 H. Saito, K. Takai and G. Yamauchi, Surf. Coat. Int., 1997, 80, 168-171.

34 F. Arianpour, M. Farzaneh and S. A. Kulinich, Appl. Surf. Sci., 2013, 265, 546-552. 